\title{
MODELOS DE SIMULAÇÃO DE ABANDONO: ESTUDO DAS FERRAMENTAS DO PATHFINDER
}

\begin{abstract}
Daniel P. Andrade ${ }^{1}$
Ramon B. Fernandes ${ }^{2}$

Maria Caroline P. B. de Araújo $^{3}$

Jassira R. P. Alves ${ }^{4}$

Luana L. G. da Silva ${ }^{5}$

\section{RESUMO}

Diante da quantidade de modelos de simulação disponíveis e variadas opções de utilização, potencialidades e restrições que tais modelos apresentam, este estudo busca como objetivo discutir parte das ferramentas e potencialidades do software Pathfinder do fabricante estadunidense Thunderhead Engineering. A discussão destas potencialidades é realizada por meio de uma revisão de literatura sobre os modelos de simulação e sobre os tipos de simulações computacionais existentes e, após isso, é apresentada uma visão geral do software Pathfinder e a discussão de suas possibilidades através de um estudo de caso em uma edificação hipotética de uso educacional. A partir do estudo de caso são extraídas as informações da simulação, demonstradas como são apresentadas na interface do software e discutidas as suas variações, aspectos positivos e negativos. O estudo de caso também contemplou um cenário que considera o uso do elevador, sendo possível demonstrar como o software permite a simulação desta situação específica. Com este estudo foi possível entender as diferenças entre os dois principais modos de simulação do software (Society of Fire Protection Engineers - SFPE e Steering), bem com outras interfaces e possibilidades, como os arquivos resumo de dados (Summary), os filtros de densidades e os gráficos com a evolução da saída de ocupantes em função do tempo.
\end{abstract}

Palavras-chave: Segurança contra incêndio; Abandono de edificações; Simulação computacional; Modelos de simulação; Pathfinder.

\footnotetext{
${ }^{1}$ Arquiteto e Urbanista (UFRN). Engenheiro de Segurança do Trabalho (UnP). Doutor em Arquitetura e Urbanismo (UFRN). Professor do curso de Arquitetura Urbanismo da UFERSA e líder do grupo de pesquisa Alpendre - Arquitetura e Representação.

${ }^{2}$ Acadêmico do curso de Arquitetura e Urbanismo da UFERSA e bolsista do grupo de pesquisa Alpendre - Arquitetura e Representação.

${ }^{3}$ Acadêmica do curso de Arquitetura e Urbanismo da UFERSA e bolsista do grupo de pesquisa Alpendre - Arquitetura e Representação.

${ }^{4}$ Acadêmica do curso de Arquitetura e Urbanismo da UFERSA e discente voluntária do grupo de pesquisa Alpendre - Arquitetura e Representação.

${ }^{5}$ Acadêmica do curso de Arquitetura e Urbanismo da UFERSA e discente voluntária do grupo de pesquisa Alpendre - Arquitetura e Representação.
} 


\title{
EVACUATION MODELS: REVIEW OF PATHFINDER'S TOOLS
}

\begin{abstract}
Given the number of simulation models available and the varied options, potentials, and restrictions that these models present, this study search to discuss part of the tools and potentialities of the Pathfinder software from Thunderhead Engineering. The discussion of these potentialities is realized through a literature review on the simulation models and the types of computer simulations. After that, is presented an overview of the Pathfinder software and the discussion of its possibilities through a case in a hypothetical building for educational use. The simulation information is extracted from the case, demonstrated as it is presented in the software interface and discussed its variations, positive and negative aspects. The case also looked on a scenario that considers the use of the elevator, being possible to demonstrate how the software allows the simulation of this specific situation. With this study it was possible to understand the differences between the two simulation modes of the software (Society of Fire Protection Engineers - SFPE and Steering) and other interfaces and possibilities, such as summary data files, density filters and graphs with the evolution of the output of occupants as a function of time.
\end{abstract}

Keywords: Fire safety; Evacuation; Computer simulation; Simulation models; Pathfinder. 


\section{INTRODUÇÃO}

Como forma de investigar as diversas condições de proteção contra incêndio, os modelos de abandono foram criados, oferecendo variados recursos e dados úteis para predição das situações de emergência, tais como simular a interação do usuário com a edificação, sinalização de emergência, e etc., para predição das situações de emergência com fogo, fumaça e outros aspectos. No entanto, considerando que esses modelos simulam de forma simplificada acontecimentos reais, durante a análise podem surgir diferentes premissas para chegar no resultado desejado, dentro da margem de segurança. Segundo Gwynne \& Kuligowski (2017), as ferramentas que podem ser consideradas como modelos de abandono são: as ferramentas de simulação computacional, o cálculo de engenharia à mão ${ }^{6}$, os exercícios de abandono, a regulamentação prescritiva e a compreensão da perspectiva do comportamento dos evacuados.

Para alcançar um resultado próximo da realidade, as simulações no meio computacional surgiram para estudar soluções mais eficientes do que os cálculos de engenharia feitos à mão. Os cálculos à mão ignoram situações comuns que podem ocorrer no processo de abandono como a alta densidade de ocupantes nas saídas, as interações entre indivíduos, as condições estruturais da construção, os efeitos do fogo e os processos de tomada de decisão dos indivíduos (KULIGOWSKI et al., 2010).

Os diversos modelos de abandono são categorizados de acordo com seus métodos utilizados e a forma com que o software analisa os dados inseridos. Segundo Kuligowski et al. (2010) os modelos são subdivididos nas três categorias: modelos de comportamento (podem abranger comportamentos mais complexos dos ocupantes, questões cognitivas e interação com o

\footnotetext{
${ }^{6}$ Tradução livre de Gwynne \& Kuligowski (2017). Entende-se que os autores se referem ao método de cálculo conforme as prescrições de normas técnicas.
} 
ambiente, outros usuários, sinalização, fogo e fumaça), modelos de movimento (relacionam-se apenas com o deslocamento dos ocupantes, assemelhando-se a um modelo de deslocamento de fluídos) e modelos de comportamento parcial (permitem inserir alguns aspectos comportamentais mais simples que influenciarão no movimento).

No presente estudo, é proposta a discussão de algumas funcionalidades do software Pathfinder (versão 2019). O software é um simulador de abandono baseado em agentes que permite avaliar a movimentação de ocupantes em um dado espaço, procurando analisar comportamentos e interações entre ocupantes. Tal ferramenta é categorizada como um modelo de comportamento parcial, sendo levadas em considerações as características de tempo de prémovimento, variação de movimento, aceleração, velocidade e alternância na escolha da saída mais próxima. No entanto o modelo parcial não simula questões psicológicas mais complexas e a interação do usuário com fogo e fumaça.

Ainda é importante lembrar que as simulações de abandono realizadas são baseadas em dados prescritos por normas que informam uma aproximação do nível de segurança necessária para cada cenário de emergência. As metodologias utilizadas pelo software servem para quantificar o tempo requerido para um abandono seguro (RSET - Required Safe Escape Time) e a margem de segurança apropriada. Podendo representar variados aspectos do processo abandono, o modelo é configurado a partir do cenário desejado, com modelagens e visualizações em $2 \mathrm{D}$ e $3 \mathrm{D}$ e cada ocupante possui seu comportamento e objetivos baseados nos dados inseridos.

Com isso, através desse trabalho, pretende-se discutir o embasamento teórico dos tipos de modelos de simulação de abandono, abordando especificamente o Pathfinder, apontando suas características e limitações para uso em análises de segurança contra incêndio em edificações. Através do software Pathfinder, foram realizadas simulações de uma mesma edificação com cenários com modos de movimentação distintos, com o intuito de 
descrever o desempenho do modelo em diferentes situações, comparar os tempos de abandono e a forma com que o modelo expõe os resultados obtidos.

\section{MODELOS DE SIMULAÇÃO DE ABANDONO}

Das formas mais elementares de que se tem conhecimento em segurança contra incêndio, antes de tudo, a função de proteção contra os efeitos indesejáveis e as dinâmicas de evacuação exemplificam uma necessidade de incorporar novos métodos de verificação e predição das ações dos usuários das edificações. Assim, tais análises de movimentação podem introduzir novas proposições e identificar soluções baseadas nas condições ambientais das diversas situações. De acordo com Pereira \& Popovic "as medidas de proteção contra incêndio contribuirão para auxiliar no salvamento das pessoas. São elas: boas saídas de emergência para facilitar a retirada dos ocupantes e o acesso dos brigadistas e integrantes do Corpo de Bombeiros.".

Para isso, os modelos de abandono têm se tornado protagonista nos estudos a fim de validar informações quanto ao comportamento de ocupantes e sua interação com a estrutura física dos edifícios em cenários de emergência. Os modelos são executados em computadores e podem simular a movimentação de ocupantes durante a evacuação e calcular o tempo final de evacuação. No entanto, como anteriormente enfatizado por Gwynne \& Kuligowski (2017), várias ferramentas podem ser designadas como modelos, sendo qualquer método que quantifique, entenda, descreva, restringe, explica, avalia ou influencia o comportamento de ocupantes na evacuação. As ferramentas que podem ser consideradas como modelos de abandono, segundo Gwynne \& Kuligowski (2017), são:

- Ferramenta de simulação computacional: este modelo é implementado em um software computacional, sendo inseridas algumas informações que representem as características físicas da população e seu comportamento baseado em ações pré-inseridas no modelo em 
frações de tempo. É necessário conhecimento do funcionamento do software para facilitar na clareza dos resultados para não os tornar limitados quanto à abordagem dos detalhes;

- Cálculo de engenharia à mão: uso de fórmulas que descrevem a movimentação e fluxo dos ocupantes no edifício. Essa metodologia muitas vezes é incorporada em ferramentas computacionais por focar principalmente no desempenho físico dos ocupantes;

- Exercícios de abandono: são práticas reais, pré-divulgadas ou não, para avaliar o desempenho do tempo de abandono no edifício. É utilizada a própria população do edifício, realizando todos os procedimentos de segurança. No entanto, esses exercícios possuem limitações por excluírem fatores ambientais nocivos, populações com conhecimento prévio do evento ou predisposta a se comportar de maneira específica, além das dificuldades de analisar diversos cenários;

- Regulamentação prescritiva: se trata das normas e instruções técnicas que devem ser executadas ainda na etapa projetual do edifício. Toda prescrição regulatória está baseada, de forma intrínseca, em dados de um modelo de evacuação. Contendo diversas questões como largura e capacidade de saídas e escadas, distância máxima percorrida, sinalização, quantidade de saídas e outros;

- Compreensão da perspectiva do comportamento individual: procura entender as expectativas e resultados do funcionamento do processo de abandono, coletando dados dos eventos através de entrevistas, experiências, reportagens e gravações. No entanto pode levantar premissas generalizadas e errôneas quanto ao comportamento das pessoas.

Diante dos fatores que favorecem a severidade de um incêndio, pode-se enfatizar a atividade desenvolvida no prédio, a forma do edifício, as condições de ventilação proporcionadas no ambiente, as propriedades térmicas dos materiais e ainda o tamanho, porque assim será possível indicar quais medidas 
cabíveis para obter os resultados satisfatórios. Tendo em vista que em ambientes mais simples será suficiente o uso de normas prescritivas, mas em lugares mais complexos, onde comporta uma população maior o uso de modelos computacionais para simular o abandono é o mais apropriado. A respeito disto, Silva (2014, p. 32) afirma que "a inclusão de medidas de proteção e combate ao incêndio e, principalmente, de meios que permitam o rápido abandono dos ambientes em chamas deve ser conscientemente analisada pelo projetista e pelo proprietário, levando em conta as condições específicas da obra, como porte da edificação, número de usuários e tipo de utilização".

As medidas de segurança para uma simulação de abandono serão definidas de acordo com a análise da arquitetura da edificação, verificando sua área total construída, altura e destinação final. Dessa maneira é que será possível enquadrar no regulamento Estadual e "conhecer as exigências relativas aos sistemas a serem implantados em razão da definição de risco de incêndio" (GOMES, 1998, p. 38). Podendo ser alcançado por meio do uso do plano de evasão para propiciar condições seguras de saída do local, possibilitada pelo uso das medidas preliminares tanto de planejamento quanto de divulgação e pelos programas de treinamento que são realizados por meio de exercícios simulados.

Por isso é de suma importância que os modelos computacionais de comportamento consigam prever as ações dos ocupantes por meio de simulação e sejam capazes de determinar o tempo necessário para uma evacuação completa e em segurança (KULIGOWSKI, 2013). É com a aplicação do plano de evasão que poderá ser propiciado condições para uma saída segura do local, possibilitada pela tomada de medidas preliminares tanto de planejamento quanto de divulgação e pelos programas de treinamento que são realizados com exercício simulados. Assim como dito por Camilo Junior (1999, p.96) ao afirmar que o Plano de Abandono funciona como um "conjunto de medidas, normas e ações estabelecidas pelo departamento de segurança da 
empresa, visando a remoção rápida, segura e eficiente de toda a população fixa e flutuante da edificação em caso de uma situação de sinistro.". Para tal fim, existem alguns softwares que simulam cenários relacionados com 0 incêndio em edificações que podem ser aplicadas de diferentes maneiras, mas coincidem ao objetivar na remoção dos ocupantes de maneira eficiente e eficaz, tais como: Pathfinder, Fire Dynamics Simulator (FDS), BuildingExodus e o GridFlow.

Os modelos computacionais de abandono são categorizados, ainda, pelo método de modelagem que o software utiliza para calcular o tempo de abandono dos edifícios. De acordo com Kuligowski et al. (2010) os três grupos básicos que variam de acordos com os dados inseridos ao modelo são:

- Modelos de comportamento: Estes modelos combinam as informações do comportamento dos ocupantes e as de movimentação até o destino final em um local seguro. Estes modelos são mais completos e podem incorporar questões de tomadas de decisão de ocupantes, isto é, reações a determinadas situações de escolha de qual trajeto seguir. Além disso, podem ser apresentados cenários com a presença de fogo e fumaça no ambiente e simular situações de desmaios e morte dos indivíduos. São exemplos desse modelo os seguintes softwares: STEPS, BuildingEXODUS, Legion, MassMotion;

- Modelos de movimento: partem da situação de mover os ocupantes de um ponto a outro sem calcular princípios de comportamento humano, sendo uma população, fluxo e velocidade homogêneos. Eles podem ser úteis em demonstrações de áreas de congestionamento, enfileiramento ou engarrafamento nos espaços do edifício. Pode-se citar o Wayout como exemplo de software;

- Modelos de comportamento parcial: nestes modelos é possível calcular a movimentação dos ocupantes juntamente com o comportamento. Os comportamentos podem ser representados pela determinação de um tempo de pré-evacuação, características básicas 
dos ocupantes e ultrapassagem. No entanto, eles não simulam ações comportamentais mais complexas e tomadas de decisão. Enquadram-se nessa categoria os seguintes softwares: EXIT89, Simulex, Pathfinder, GridFlow.

Assim, os modelos de abandono têm se tornado ferramentas essenciais que ajudam no funcionamento adequado das estratégias emergenciais situações de abandono, tendo em vista que na simulação é analisado o comportamento que costuma ser esperado pelos ocupantes. De acordo com Ronchi (2020), para que os modelos reproduzam situações de comportamento humano é necessária uma abordagem multidisciplinar, diversas disciplinas desempenham um papel fundamental no desenvolvimento do modelo de abandono como a engenharia, psicologia, física, matemática, sociologia, ciência da computação e outros. No entanto, é necessário ter conhecimento de como os modelos a serem utilizados funcionam e ter clareza ao apresentar os dados, as restrições e as relativizações que o modelo apresenta.

\section{O modelo de simulação a partir do Pathfinder}

O Pathfinder é um software desenvolvido e comercializado pela empresa Thunderhead Engineering, que tem como premissa básica desenvolver simulações de abandono em situações de incêndios, as quais possibilitam a análise da movimentação da população envolvida. O programa opera através de dois métodos para modelagem da simulação de movimento, sendo esses 0 SFPE - Society of Fire Protection Engineers ${ }^{7}$ de Nelson \& Mowrer (2002) e o Steering.

O modo Steering ou de direção, padrão do Pathfinder, fundamenta-se no conceito de comportamento de direção inverso, o qual foi aprimorado por Amor et al. (2006) embasado no modelo de comportamento de direção retratado anteriormente por Reynold (1999) em "Steering Behaviors For Autonomous

\footnotetext{
${ }^{7}$ Sociedade de Engenheiros de Proteção contra Incêndios.
} 
Characters". Nesse modo de simulação é utilizado um sistema de controle de direção, fazendo com que os ocupantes mantenham uma distância regular dos outros participantes, para assim, reduzir as colisões. Além disso, deve-se salientar que as portas não atuam no controle das taxas de fluxos de ocupantes, mas decorrem da interação dos ocupantes uns com os outros e com os limites.

Quanto ao modo SFPE, que é um recurso de origem americana alternativo do software, é empregado um modelo de abandono baseado em fluxos, seguindo diretrizes que constam no "SFPE Handbook of Fire Protection Engineering" de Nelson \& Mowrer (2002) e "SFPE Engineering Guide: Human Behaviour in Fire" do SFPE (2003). Nesse modo, as portas limitam o fluxo, a velocidade de deslocamento é afetada pela densidade de ocupantes, e esses não são orientados a evitar os demais.

Uma das funcionalidades do Pathfinder é referente a sua compatibilidade com arquivos desenvolvidos em Computer-Aided Design ${ }^{8}$ (CAD), possibilitando a fácil inserção das características da edificação, como a quantidade de pavimentos e seus layouts, o quantitativo da população que a edificação comporta. Essas informações permitem diagnosticar os eventuais obstáculos que poderão prejudicar no processo de abandono, em diferentes cenários e estudar soluções projetuais para contornar os impasses apresentados, auxiliando na elaboração de um projeto que preza pela segurança das pessoas que utilizarão o edifício. A Figura 1 retrata a interface do software, onde é possível observar a disposição das plantas quando importadas e também os ocupantes, representados pelos cilindros, os quais encontram-se distribuídos pelos ambientes.

\footnotetext{
${ }^{8}$ Desenho auxiliado por computador.
} 
Revista Científica do Corpo de Bombeiros Militar de Pernambuco

Artigo Publicado no Vol.06 N.17 - Edição Jul a Dez 2020 - ISSN 2359-4829

Versão on-line disponível em: http://www.revistaflammae.com

Figura 1 - Tela principal do programa Pathfinder

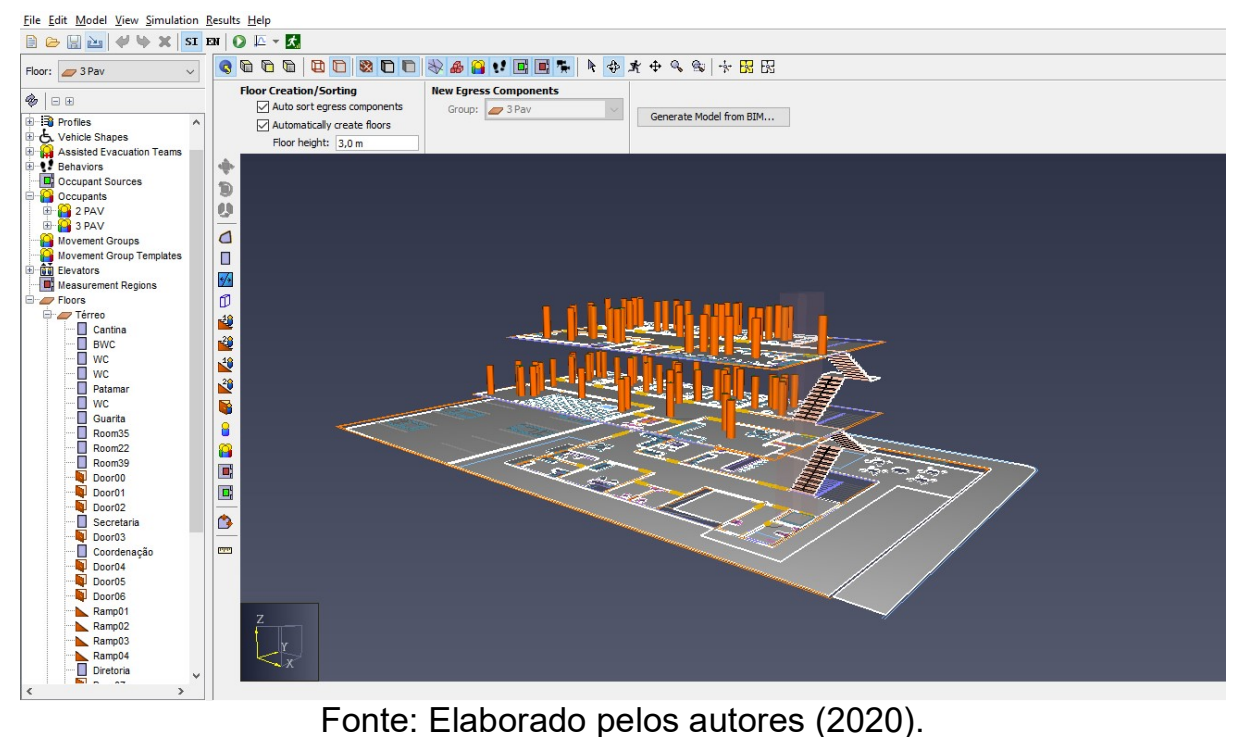

\section{Dinâmica da Simulação}

Como já citado, o Pathfinder é classificado como um modelo de comportamento parcial, fundamentado em pesquisas de observação do comportamento humano, que segundo Ono (2010), essencialmente calculam o movimento das pessoas, e incluem alguns comportamentos, como a inserção do tempo pré-movimento nos ocupantes e o comportamento de ultrapassagem. Com isso, o programa dispõe de um conjunto de modelos humanos, que possuem características diversificadas, como idade, gênero, altura e velocidade caminhamento, assim possibilitando a representação de vários grupos populacionais.

A definição do perfil dos ocupantes da edificação em estudo é um fator significativo para o desenvolvimento da simulação, pois será por meio da combinação dos parâmetros atribuídos que cada ocupante irá escolher seu trajeto até alcançar uma saída. Assim, os comportamentos são definidos de acordo com a preferência do utilizador, que pode designar ao ocupante um procedimento de espera em um local até ocorra um determinado evento, ou de busca, direcionando o ocupante a se mover até um ponto específico, podendo 
esse ser uma sala, elevador ou uma porta saída. Deve-se destacar que há também a possibilidade de mesclar diferentes comportamentos para a população, que, porventura, podem modificar-se com o passar do tempo.

Terminado a fase de incorporação das informações no modelo e executada a simulação, são obtidos os resultados, dentre os quais compreendem um arquivo de resumo, gráficos e uma visualização em 3D. 0 arquivo de resumo oferece dados acerca da densidade, do tempo de saída e entrada dos ocupantes em salas, portas ou escadas, e também em relação ao fluxo dos ocupantes. Os gráficos disponíveis permitem observar a quantidade de pessoas nos ambientes ao longo do tempo, assim como também os dados contidos no arquivo de resumo, tanto por meio de gráficos de linhas, como de contornos. Já a representação em 3D proporciona ao usuário a experiência de percorrer o modelo de maneira mais detalhada, sendo possível visualizá-lo a partir de diferentes perspectivas, bem como pausar ou adiantar a simulação.

\section{Limitações do modelo}

Conforme Araujo (2008), estudar o comportamento das pessoas em incêndios é fundamental para determinar como proceder nessas situações, apontando o trajeto a percorrer até a rota de fuga e a saída em segurança. Assim sendo, o fato de o Pathfinder constituir o grupo de modelos de comportamento parcial torna-se uma das limitações apresentadas pelo software, dado que este não prevê as diferentes reações humanas em situações adversas.

Nesse tipo de simulação não são consideradas questões cognitivas e psicológicas mais complexas dos ocupantes. De acordo com Jacobi (1999), a forma como as pessoas vão se comportar, mediante um cenário de risco, é dependente da sua concepção em relação à uma situação de perigo, que se relaciona com diversas variáveis, dentre essas, as psicológicas. Sendo assim, essas são condições consideradas relevantes na obtenção de resultados mais 
precisos e que se assemelham à realidade, as quais estão ausentes na simulação gerada pelo software.

A interação com a sinalização de emergência também é outro fator que o software não permite simular. Em situações em que a população não apresenta familiaridade com a edificação, o apoio da sinalização de emergência pode ser fator decisivo para um processo de abandono mais eficiente, principalmente em geometrias complexas.

Outras questões não integradas pelo programa, que também se correlacionam com a movimentação e o comportamento das pessoas, são os cenários na presença de fumaça ou fogo. Cabe também aqui destacar que os exemplos simulados devem ser considerados ideais, ou seja, não contam com a influência de comportamentos não adaptativos, uma vez que não são avaliados todos os riscos resultantes de um incêndio; assim como também, usufruem dos sistemas de proteção da edificação em perfeitas condições e pressupõe que todas as saídas de emergência permanecem desobstruídas durante o processo de abandono.

\section{Tempo requerido para um abandono seguro (RSET)}

Em episódios de incêndio, na maioria dos casos faz-se necessário desocupar a edificação, dado que a segurança das pessoas deve ser posta em primeiro lugar. À vista disso, considera-se que um projeto de segurança contra incêndio ideal tem como um de seus principais objetivos assegurar a disponibilidade de tempo necessário para o escape da população. Nesse contexto, a norma britânica PD 7974 - 6 (2019) determina que a predição do tempo disponível para um abandono seguro (ASET - Available Safe Escape Time) sempre deverá ser maior que o tempo requerido para um abandono seguro (RSET - Required Safe Escape Time) para que possua uma margem de segurança. 
Para determinar o tempo de abandono é essencial considerar todas as variáveis que influenciam nas respostas comportamentais dos ocupantes, dado que essas podem apresentar diferentes graus de envolvimento em todas as fases do processo de fuga. Isto posto, compreende-se que o tempo total de abandono é constituído pelo somatório de tempos parciais, que conforme Valentim (2008, p.197) "faz-se necessário entender cada uma destas parcelas de tempo, pois se a somatória de tempos que precedem o caminhamento for muito alta, quando o ocupante decidir efetivamente iniciar o movimento, o limite tolerável pode ser mínimo".

Segundo a PD 7974 - 6 (2019) as etapas que compõem o tempo requerido para um abandono seguro são: o tempo de ignição e detecção; tempo de detecção até o acionamento de um aviso geral aos ocupantes, como o alarme de emergência; tempo de evacuação composto pelo, tempo de prémovimento e o tempo do trajeto até o local seguro (Figura 2).

Figura 2 - Variáveis que compõe o tempo de abandono seguro

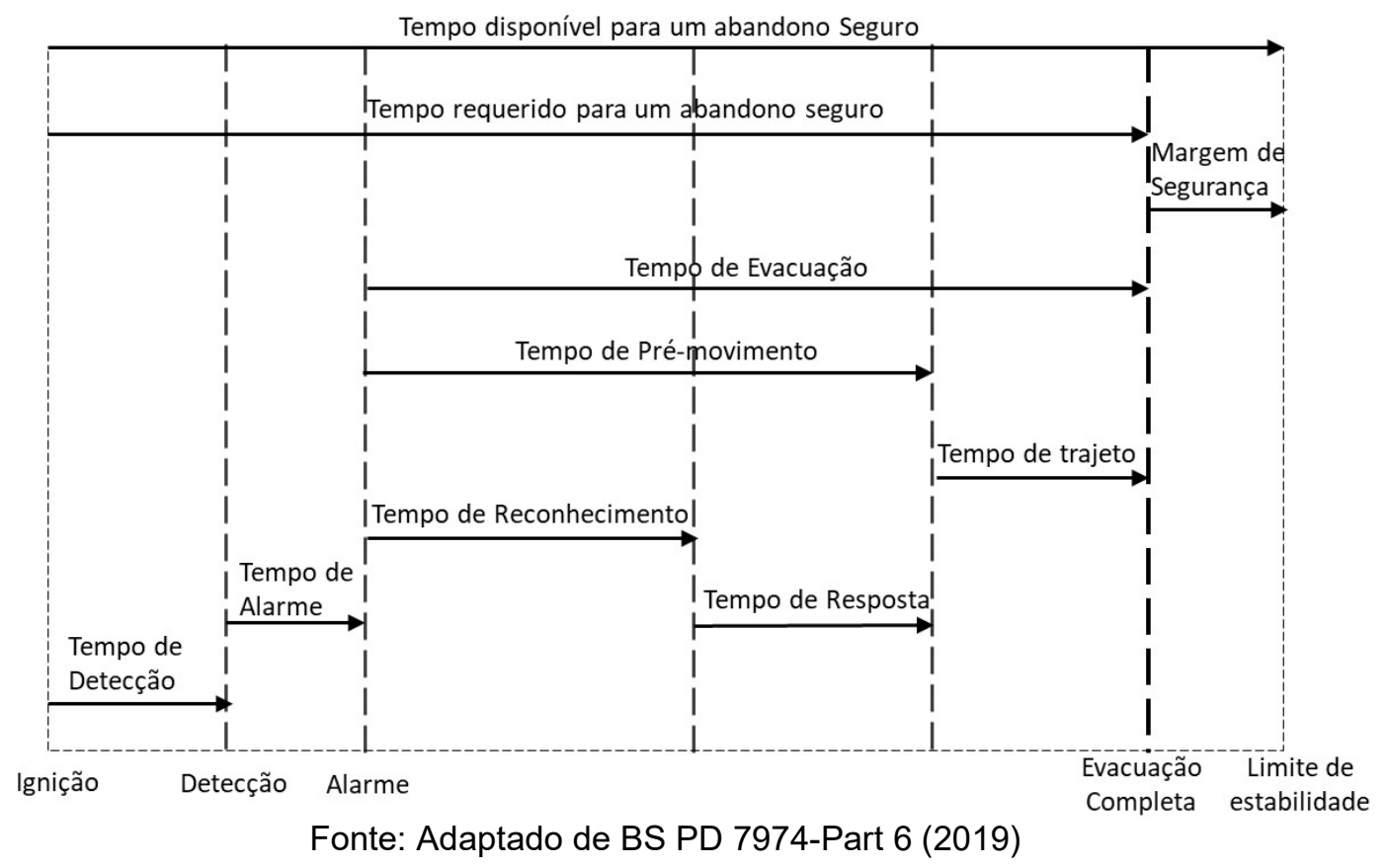




\section{ESTUDO DE CASO}

Optou-se por realizar um estudo de caso sobre o software Pathfinder, a partir da simulação de uma situação hipotética com a finalidade de apresentar parte das suas ferramentas, possibilidades e limitações.

\section{Desenvolvimento da modelagem em análise}

Como forma de avaliar o desempenho do software Pathfinder, considerando os recursos anteriormente demonstrados, foi elaborado um cenário hipotético simulado em um edifício de três pavimentos de uso educacional. Além disso, as simulações são designadas para obter informações quanto a movimentação dos usuários no edifício, a influência da geometria das saídas de emergência, a dinâmica de evacuação e o tempo obtido. O edifício contém um total de oito salas de aula, um auditório, sala de professores, biblioteca e outros. Uma planta correspondente ao segundo pavimento é demonstrada na Figura 3 como maneira de apresentar a configuração interna da edificação em estudo. A área total de cada pavimento varia de $203 \mathrm{~m}^{2}$ à $245 \mathrm{~m}^{2}$. 


\section{Revista FLAMMAE}

Revista Científica do Corpo de Bombeiros Militar de Pernambuco

Artigo Publicado no Vol.06 N.17 - Edição Jul a Dez 2020 - ISSN 2359-4829

Versão on-line disponível em: http://www.revistaflammae.com

Figura 3 - Planta baixa do segundo pavimento da edificação em estudo

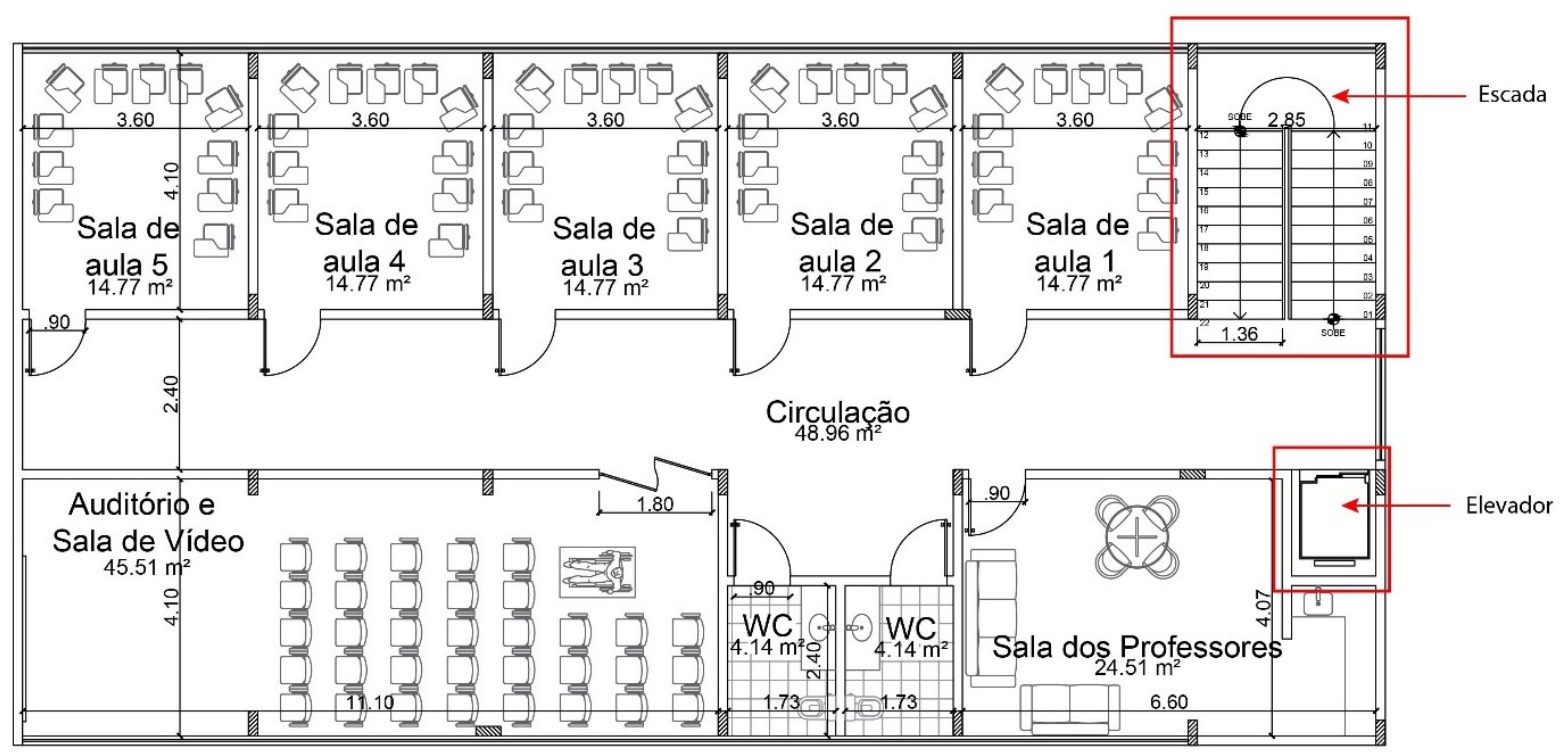

Fonte: Elaborado pelos autores (2020).

Para este estudo foram investigados os dois modos de movimentação apresentados pelo programa, o Steering e o SFPE. Em ambos os cenários se adotou a população correspondente ao recomendado na Instrução Técnica No11 (IT - No11) da Polícia Militar de São Paulo (PMESP) - IT - No11 (PMESP, 2019). A edificação foi enquadrada como sendo do grupo E-1 (Escolas em geral) sendo aplicado os parâmetros devidos para o cálculo, no qual é apresentado a capacidade de uma pessoa por $1,50 \mathrm{~m}^{2}$ de área de sala de aula, de acordo com a IT - №11 (PMESP, 2019). Assim, para este estudo foi aplicada a população no total de 88 ocupantes para os dois cenários.

Para determinar o tempo de pré-movimento, foram utilizadas as informações fornecidas pela norma britânica PD 7974 - 6 (2019), devido à falta de uma referência nacional para avaliar este parâmetro. Seu principal objetivo é considerar na simulação o tempo que os ocupantes levam para ter ciência da ocorrência e iniciar seu processo de abandono. Com isso, o tempo de prémovimento a ser considerado foi de $30 \mathrm{~s}$ a $1 \mathrm{~min}$ e $30 \mathrm{~s}$, sendo subdividido de forma aleatória entre os ocupantes. Este critério está baseado na gestão da cultura de treinamentos para abandono dos ocupantes, um bom padrão de 
treinamento e gestão de segurança contra incêndio. A partir disso, o abandono a ser simulado é considerado como sendo ordenado e treinado. Sabe-se que esta nem sempre é uma situação usual, principalmente em escolas, porém esta relativização precisou ser feita de forma a se adequar às limitações que o software impõe. No Quadro 1 são apresentados os dados inseridos no modelo em análise.

Quadro 1 - Dados de comportamento inseridos no
\begin{tabular}{|l|c|}
\hline \multicolumn{2}{|c|}{ DADOS BÁSICOS DE COMPORTAMENTO } \\
\cline { 2 - 3 } Tempo de Alarme & $0 \mathrm{~s}$ \\
\hline Tempo de pré-movimento & $30 \mathrm{~s}-1 \mathrm{~min} \mathrm{e} 30 \mathrm{~s}$ \\
\hline Velocidade Máxima & $1,19 \mathrm{~m} / \mathrm{s}$ \\
\hline Altura dos Ocupantes & $1,54 \mathrm{~m}-1,83 \mathrm{~m}$ \\
\hline
\end{tabular}

Fonte: Elaborado pelos autores (2020).

Com isso, os ocupantes são adicionados de forma dispersa nos ambientes de maior uso da população, neste caso nas salas de aula, e são padronizados pelos perfis e comportamentos. Para definir o comportamento dos ocupantes, além de determinar o tempo de pré-movimento, foi indicado que os mesmos deveriam sair dos respectivos compartimentos e procurar a saída mais próxima, sendo direcionados até o pavimento de descarga no térreo em um local seguro localizado na área externa do edifício. Na planta são dispostas duas opções de saída, as escadas e o elevador localizadas na lateral direita da edificação. Para esse estudo, foi considerado que o elevador é apropriado para realizar o abandono seguro de ocupantes. Embora não seja comum em edificações deste porte e altura o uso de elevadores para fins de abandono, optou-se pelo uso do mesmo como maneira de demonstrar a funcionalidade de simular o uso do elevador que o software apresenta.

Para o modo Steering o software utiliza ainda alguns parâmetros específicos de movimento do modelo, estes estão dispostos no Quadro 2. 
Revista Científica do Corpo de Bombeiros Militar de Pernambuco

Artigo Publicado no Vol.06 N.17 - Edição Jul a Dez 2020 - ISSN 2359-4829

Versão on-line disponível em: http://www.revistaflammae.com

Quadro 2 - Parâmetros padrões para o modo Steering

\begin{tabular}{|l|l|}
\hline \multicolumn{2}{|c|}{ PARÂMETROS ESPECíFICOS DO MODO STEERING } \\
\hline $\begin{array}{l}\text { Tempo de Aceleração (Especifica a quantidade de tempo que os ocupantes } \\
\text { levam para alcançar a velocidade máxima a partir do repouso ou repousar após } \\
\text { alcançar a velocidade máxima) }\end{array}$ & $\begin{array}{l}\text { Padrão }= \\
1,1 \mathrm{~s}\end{array}$ \\
\hline $\begin{array}{l}\text { Tempo de Persistência (Quantidade de tempo que um ocupante manterá um } \\
\text { nível elevado de prioridade ao tentar resolver algum conflito de movimentação) }\end{array}$ & $\begin{array}{l}\text { Padrão }= \\
1,0 \mathrm{~s}\end{array}$ \\
\hline $\begin{array}{l}\text { Tempo de Resposta à Colisão (Controla a distância que o ocupante começará a } \\
\text { registrar ao colidir com outros ocupantes) }\end{array}$ & $\begin{array}{l}\text { Padrão }= \\
1,5 \mathrm{~s}\end{array}$ \\
\hline $\begin{array}{l}\text { Fator de Demora (Especifica a fração de velocidade dos ocupantes que eles são } \\
\text { considerados lentos) }\end{array}$ & $\begin{array}{l}\text { Padrão }= \\
0,1 \mathrm{~s}\end{array}$ \\
\hline $\begin{array}{l}\text { Distância de Conforto (Distância necessária que um ocupante tentará manter } \\
\text { dos outros em uma fila) }\end{array}$ & $\begin{array}{l}\text { Padrão }= \\
0,08 \mathrm{~m}\end{array}$ \\
\hline
\end{tabular}

Fonte: Elaborado pelos autores (2020).

A partir disso, as simulações para o cenário 01 (modo SFPE) terminaram em 2 min $41 \mathrm{~s}$ e para o cenário 02 (modo Steering) em 2min56s. Com os resultados obtidos percebe-se que o tempo de abandono entre os dois modos apresenta-se bastante semelhante, havendo apenas uma diferença de $15 \mathrm{~s}$ entre esses. Contudo, para o presente estudo torna-se pertinente verificar alguns aspectos exibidos em ambas simulações, a fim de compreender a dinâmica de abandono em cada uma. No Quadro 3 estão dispostos os tempos de abandono, em segundos, extraídos do arquivo Summary gerado pelo programa para os dois cenários, sendo eles o tempo mínimo que o primeiro ocupante alcançou a porta de saída na área externa do edifício, o tempo máximo ou tempo total e o tempo médio.

Quadro 3 - Tempos de abandono de acordo com cada modo de movimentação

\begin{tabular}{|l|l|l|}
\hline Tempos de abandono (s) & SFPE & Steering \\
\hline Mínimo & 71 & 73 \\
\hline Máximo & 161,6 & 176,1 \\
\hline
\end{tabular}




\begin{tabular}{|l|l|l|}
\hline Médio & 123,1 & 129 \\
\hline
\end{tabular}

Fonte: Elaborado pelos autores (2020).

Ainda que o tempo de pré-movimento considerado compreenda um intervalo de $30 \mathrm{~s}$ à $90 \mathrm{~s}$, percebeu-se que na simulação utilizando o modo SFPE, as pessoas começam a movimentar-se por volta dos $35 \mathrm{~s}$, sendo que o primeiro ocupante alcança a porta de saída após $71 \mathrm{~s}$ decorridos do início da simulação, conforme mostrado no arquivo de resumo que o programa fornece (Quadro 3). Com isso, foram também destacados alguns tempos de abandono específicos, e, respectivamente, o número de pessoas que já haviam deixado a edificação naquele instante (Quadro 4), com propósito de realizar um comparativo com o exibido pelo outro recurso de movimentação.

Quadro 4 - Número de pessoas que saíram em função do tempo decorrido para o modo SFPE

\begin{tabular}{|l|l|l|l|l|l|}
\hline Tempo (s) & 60 & 90 & 120 & 150 & 161,8 \\
\hline$N^{\circ}$ de pessoas que saíram & 0 & 10 & 39 & 72 & 88 \\
\hline Percentual (\%) & 0 & 12 & 45 & 82 & 100 \\
\hline
\end{tabular}

Fonte: Elaborado pelos autores (2020).

Corroborando com as informações acima, o gráfico apresentado na Figura 4 possibilita aferir o tempo total de abandono, o qual denota a quantidade de ocupantes que saem à medida que o tempo decorre. Esse gráfico é uma ferramenta útil, pois permite uma análise tanto de componentes individuais, dado que apresenta informações acerca das pessoas que abandonaram salas, ou cruzaram portas e escadas, como também especifica o quantitativo da população que sai da edificação durante o tempo total da simulação, sendo esse último o abordado para o estudo. Através dele é 
possível notar, por exemplo, em que espaço temporal da edificação houve congestionamentos ou atrasos na saída dos ocupantes. Por meio deste, constata-se uma taxa de abandono de aproximadamente 1 pessoa a cada $2 \mathrm{~s}$.

Figura 4 - Gráfico com a evolução da saída dos ocupantes em função do tempo

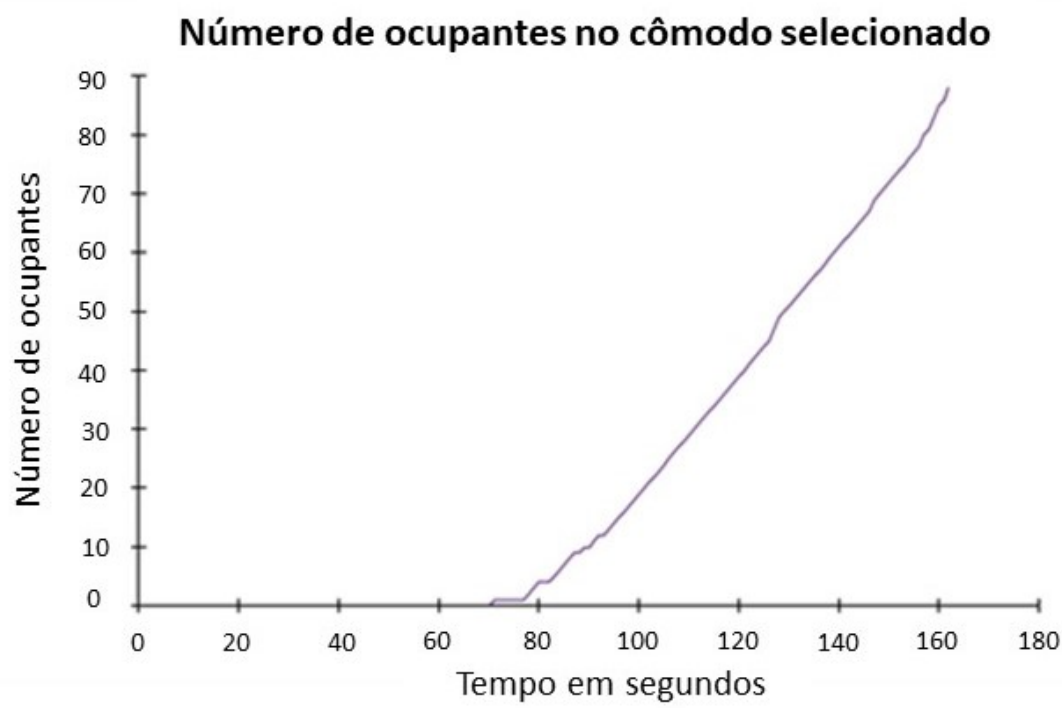

Fonte: Elaborado pelos autores, 2020.

Além do tempo de abandono, a simulação também concede informações sobre áreas de possíveis riscos de congestionamento, as quais tornam-se úteis para minimizar as consequências de qualquer incidente indesejado durante $o$ processo de fuga. Nesse contexto, para compreender os pontos de congestionamento faz-se a utilização dos mapas de densidade. A Figura 5 apresenta os mapas de densidade observados no modo SFPE, no tempo de 01 min e $37 \mathrm{~s}$, dos três pavimentos da edificação em estudo. $\mathrm{O}$ gradiente de cores exibe a densidade de ocupantes $/ \mathrm{m}^{2}$, que varia de 0,55 a 3,00 , sendo representadas por zonas frias e quentes, respectivamente. Assim, verifica-se que o ponto em que há maior concentração de pessoas é a escada do segundo pavimento, onde a densidade alcança sua máxima.

Figura 5 - Mapas de densidade do $1^{\circ}(A), 2^{\circ}(B)$ e $3^{\circ}(C)$ pavimento no modo SFPE 


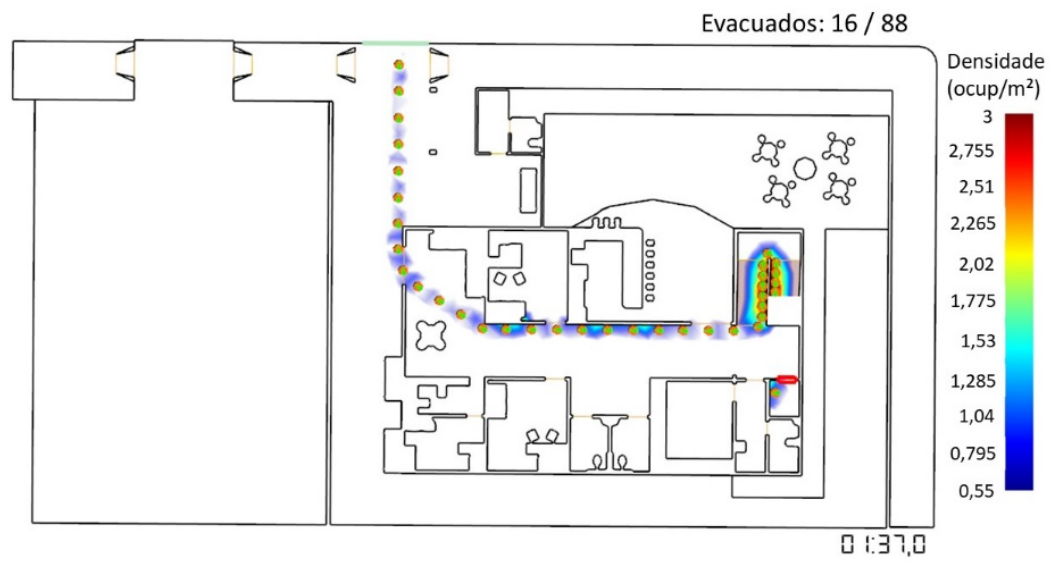

(A)

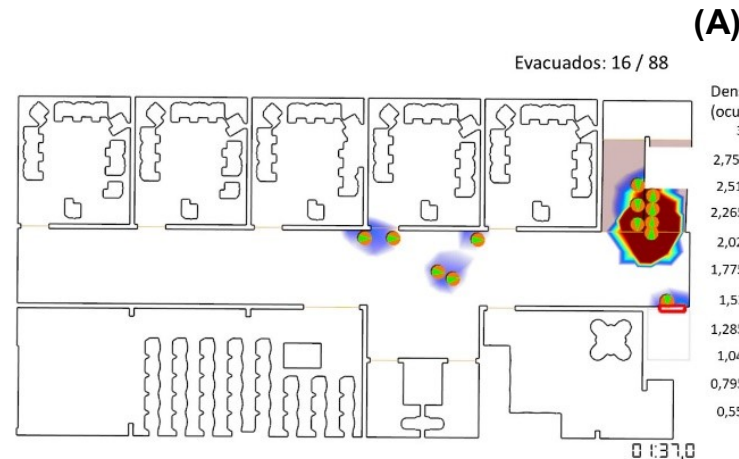

(B)

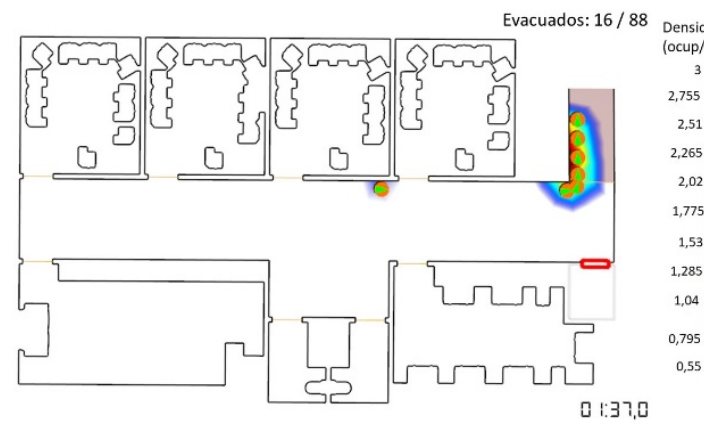

(C)

Fonte: Elaborado pelos autores (2020).

Por outro lado, os resultados do segundo cenário, utilizando o modo Steering, se mostraram semelhantes ao anterior, porém com algumas especificidades. A simulação mostrou um tempo total de evacuação de 2 min e $56 \mathrm{~s}$, apenas $15 \mathrm{~s}$ segundo a mais que a evacuação em modo SFPE (evacuação em fila). Isso demonstra que, o aglomerado de pessoas em torno das saídas pode aumentar o tempo de abandono do local em um cenário real.

Para este cenário, também foi utilizado o tempo de pré-movimento estando entre $30 \mathrm{~s}$ a $90 \mathrm{~s}$, a partir disso, no modo Steering, apenas por volta dos $33 \mathrm{~s}$ os ocupantes iniciam sua movimentação e aos $73 \mathrm{~s}$ o primeiro ocupante alcança o local seguro fora do edifício. Diferentemente do modo SFPE, neste cenário os ocupantes procuram a menor distância para alcançar a saída, sendo o mais próximo do real, além disso levam em conta as colisões 
entre ocupantes e obstruções, sem admitir que os ocupantes ocupem o mesmo espaço. No Quadro 3, anteriormente apresentado, é possível observar os tempos de abandono que o programa fornece através do arquivo resumo. Além disso, como forma de aferir os instantes de tempo, a quantidade de pessoas e o percentual de abandono com os do cenário anterior, o Quadro 5 destaca tais aspectos.

Quadro 5 - Número de pessoas que saíram em função do tempo decorrido para o modo Steering

\begin{tabular}{|l|l|l|l|l|l|}
\hline Tempo (s) & $\mathbf{6 0}$ & $\mathbf{9 0}$ & $\mathbf{1 2 0}$ & $\mathbf{1 5 0}$ & $\mathbf{1 7 6 , 3}$ \\
\hline $\mathrm{N}^{\circ}$ de pessoas que saíram & 0 & 8 & 32 & 65 & 88 \\
\hline Percentual (\%) & 0 & 10 & 37 & 74 & 100 \\
\hline
\end{tabular}

Fonte: Elaborado pelos autores (2020).

Como no cenário anterior, foi extraído o gráfico que informa a relação de número de ocupantes e o tempo em segundos durante o abandono, podendo ser analisado o desempenho gradual do processo e a quantidade de ocupantes evacuados total no instante de tempo final. O gráfico se apresentou similar ao modo SFPE, com um abandono próximo a linearidade e sem grandes variações no fluxo de saída, obtendo uma taxa de abandono de 1 pessoas a cada $2 \mathrm{~s}$.

Além disso, foram extraídos os mapas de densidade que destacam os possíveis pontos de congestionamento de pessoas por metro quadrado no processo de abandono. Assim, a Figura 6 apresenta os mapas de densidade extraídos do segundo cenário, no modo Steering, no tempo de 1 min e $37 \mathrm{~s}$, dos três pavimentos da edificação em análise. Sua taxa de densidade (ocupantes $/ \mathrm{m}^{2}$ ) varia de 0,55 a 3,00 , estando representada pelas cores em gradiente. Neste caso, o ponto de maior concentração de ocupantes continua sendo o segundo pavimento onde os níveis atingem sua variação máxima, no hall de espera das escadas, isto se deve à espera dos ocupantes do pavimento 


\section{Revista FLAMMAE}

Revista Científica do Corpo de Bombeiros Militar de Pernambuco

Artigo Publicado no Vol.06 N.17 - Edição Jul a Dez 2020 - ISSN 2359-4829

Versão on-line disponível em: http://www.revistaflammae.com

para continuarem seu trajeto pelas escadas e o acúmulo dos demais provenientes do pavimento superior.

Figura 6 - Mapas de densidade do $1^{\circ}(\mathrm{A}), 2^{\circ}(\mathrm{B})$ e $3^{\circ}(\mathrm{C})$ pavimento no modo Steering

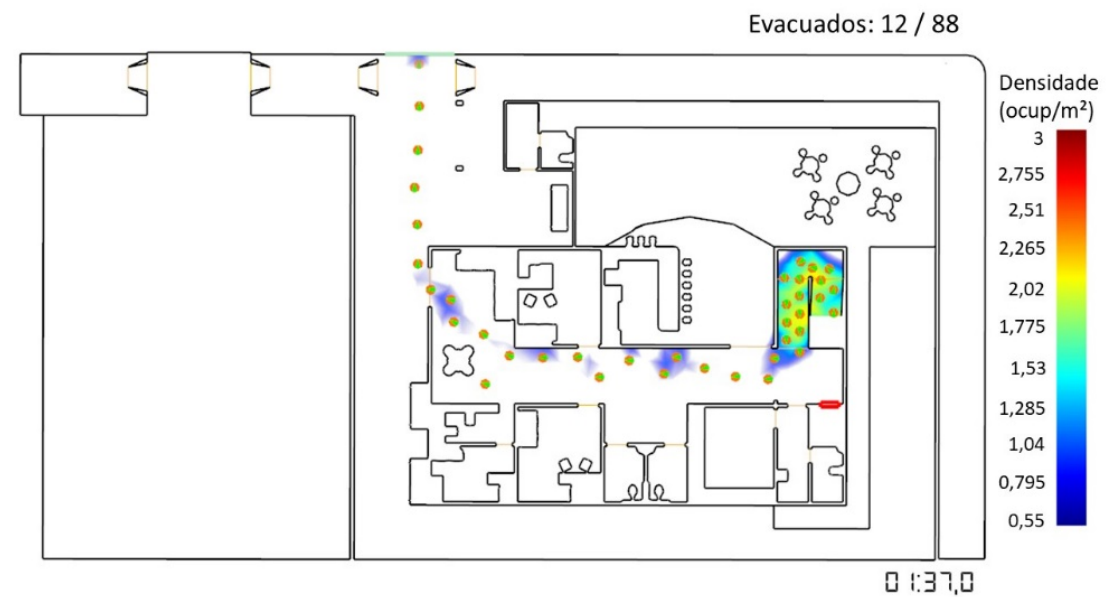

(A)

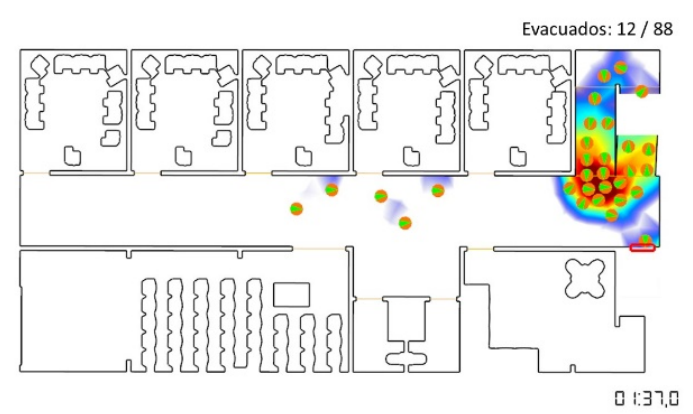

(B)

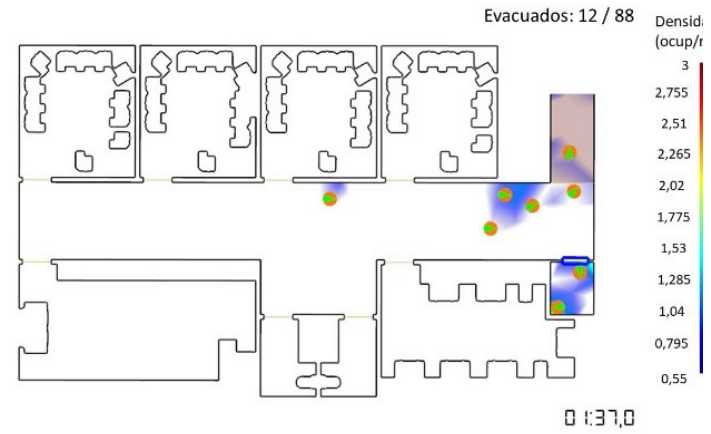

(C)

Dentre os recursos disponibilizados pelo Pathfinder tem-se ainda a possibilidade de inserir nas simulações o elevador de emergência, possibilitando análises do desempenho da edificação com a presença de diferentes saídas. Juntamente a isso, a simulação permite observar o comportamento dos ocupantes, em relação a alternância na escolha das saídas.

Considerando cenários em que se emprega o elevador de emergência, faz-se importante salientar alguns fatores que irão influenciar a escolha da população em selecioná-lo para compor sua rota de fuga, como o tempo de espera, altura do edifício e educação quanto ao uso em situações de 
emergência. Assim, para este estudo foi adotado o uso do elevador tendo como premissa que o mesmo é apropriado para situações de emergência e não apenas um elevador qualquer, apesar de que segundo a IT - No11 (PMESP, 2019) a edificação não necessita de elevador de emergência devido o grupo ao qual está enquadrada e a sua altura.

Conforme Levin \& Groner (1995) o tempo de espera dos ocupantes até a chegada do elevador tende a ser menor que os atrasos tolerados durante 0 abandono por escadas, isso deve-se à preocupação em relação ao funcionamento do elevador.

Relativo à altura, sabe-se que essa torna-se um aspecto determinante para que os ocupantes recorram ao elevador, pois conforme aumenta o nível do pavimento, maior serão as dificuldades de abandono, pois, consequentemente, aumenta as distâncias a serem percorridas e exige mais esforço físico.

A instrução dos ocupantes também irá atuar na tomada de decisão, e atentando para o fato de que comumente os elevadores possuem indicação de não utilização em situação de incêndio, a população estará propensa a rejeitar o uso dessa rota, mesmo que seja destinada ao abandono. Segundo um estudo realizado por Zmud (2007, apud REIS, 2011, p. 21) "16\% dos ocupantes do edifício acredita que era raramente seguro usar um elevador, e $3 \%$ dos ocupantes do edifício acreditava que normalmente era seguro usar um elevador para evacuar em um incêndio".

Como a planta da edificação utilizada para estudo apresentava um elevador, optou-se por utilizá-lo também como um meio de abandono, para ilustrar aplicação da ferramenta do programa. A cabine do elevador no projeto analisado possui as seguintes dimensões: $1,11 \mathrm{~m}$ de largura e 1,23 m de profundidade, e uma capacidade de 6 pessoas. O programa apresenta como possibilidade a escolha da velocidade do elevador a depender do cenário desejado, como maneira de se adequar a uma situação real e os diversos tipos possíveis de elevador. Ao simular o cenário em um modo padrão, destinando o 
comportamento para que os ocupantes procurassem a saída mais próxima, percebeu-se que esses só utilizavam o elevador quando o hall da escada estava com alta densidade de ocupantes. Este comportamento se deve à uma das configurações atribuídas no perfil dos ocupantes, chamada de tempo de espera do elevador, que especifica o tempo que o ocupante prefere utilizar o elevador. Quando esse tempo acaba, é mais provável que o ocupante opte pelo uso das escadas. Com isso, amparado nos argumentos descritos no estudo de Zmud (2007, apud REIS, 2011, p. 21) anteriormente apresentados, foi estimado na simulação para que cerca de $3 \%$ dos ocupantes utilizassem o elevador nos cenários em análise.

Após observar os resultados da simulação, foi possível constatar que a utilização do elevador se deu como programado, uma vez que apenas uma parcela da população foi destinada a usar o elevador. $O$ arquivo resumo da simulação mostra que dos 88 ocupantes, apenas três, ou seja, $4 \%$ da população optou por usar o elevador, tanto no modo SFPE quanto no Steering. A partir disso, a possibilidade de o programa simular abandono utilizando o elevador deve ser avaliado de forma particular de acordo com cada estudo realizado, visto que não se trata de uma situação comum. Portanto, recomenda-se que o cenário avaliado ocorra utilizando variáveis mais próximas do real para assim chegar a resultados mais refinados.

\section{CONSIDERAÇÕES FINAIS}

Dentro de um estudo de segurança contra incêndio em edificações, compreender o processo de retirada dos ocupantes faz-se essencial, de forma que esse pode se tornar um tema central. Nesse contexto, os modelos de simulação computacional fazem-se instrumentos bastante relevantes, visto que permitem a realização de estudos detalhados tanto em relação à ambientes específicos da edificação, e desta como um todo. Ressalta-se também que a implementação dessas ferramentas contribui na definição de soluções que 
visem à segurança dos usuários, dado que prevê questões não consideradas nas prescrições normativas.

Os modelos de simulação computacional são uma redução e simplificação da realidade, repletos de limitações e relativizações, porém ainda assim podem ser uma ferramenta útil para o desenvolvimento de projetos por conseguir captar, simular e antever alguns aspectos da realidade.

Em se tratando de tempo mínimo e máximo de abandono, deve-se ter conhecimento dos fatores que podem vir a gerar alguma interferência neste, como: o número de pavimentos do prédio, o layout, a densidade populacional, entre outros. Para o desenvolvimento deste estudo contemplou-se a abordagem do software Pathfinder, notando-se que esse apresentou uma interface intuitiva e de fácil compreensão, viabilizando a obtenção de informações de caráter quantitativo, como o tempo de escape, e também qualitativos, referente aos pontos de aglomeração. Além disso, foi possível avaliar o deslocamento dos usuários, por meio de diferentes padrões de movimentação que o software oferece.

Com isso, avaliando o uso do programa computacional Pathfinder a partir do caso estudado foi constatado que, em ambos os cenários, verificou-se um abandono constante sem afetar significativamente na segurança e integridade dos ocupantes, apesar de apresentar pequenos acúmulos no segundo pavimento, estes não interferem no processo por serem rapidamente resolvidos. Quando comparado o percentual de ocupantes evacuados, nos intervalos de tempo delineados, é notória que o modo SFPE induz a um processo mais rápido, uma vez que considera fatores pré-concebidos baseados em princípios do cálculo à mão em sua forma íntegra não estando suscetível a irregularidades de movimento.

Ainda, se faz necessário evidenciar que as conclusões aqui levantadas se baseiam nos resultados obtidos de um estudo de caso. Ressalta-se que o ambiente simulado retrata um cenário considerado ideal, e que possui suas limitações, como interações da população com fogo e fumaça. Os resultados 
obtidos demonstraram que o menor tempo foi do cenário utilizando a dinâmica de movimentação do modo SFPE que designa um abandono em fila, no entanto na execução real esta prática é dificilmente contemplada. Ao realizar o abandono no modo Steering, são utilizadas premissas que se aproximam mais do real apesar de perceber um aumento de tempo total.

Destaca-se que a utilização do programa não se restringe aos recursos abordados nesse estudo, visto que esse usufruiu de uma parcela das ferramentas ofertadas. Assim, não se limita a temática das simulações computacionais com Pathfinder a esse trabalho, de forma que se pretende em estudos futuros expandir os cenários avaliados, integrando-o com outros softwares que permitam simular incêndios com fumaça, gases tóxicos, sinais de alarme, entre outros.

\section{REFERÊNCIAS}

AHMED, S.; MEHMOOD, S.; KRISTENSEN, A. S. Critical Analysis of Computer-based Evacuation Simulation of an Educational Institution. In: Proceedings of the 11th International Conference On Computer Modeling and Simulation. 2019. p. 26-30.

AMOR, H. B.; MURRAY, J.; OBST, O. Fast, Neat and Under Control: inverse steering behaviors for physical autonomous agents. In: RABIN, S. Ai Game Programming Wisdom 3. Charles River Media Publisher, 2006.

BRITISH STANDARD INSTITUTION. PD 7974-Part 6: The application of fire safety engineering principles to fire safety design of buildings - Part 6: Human factors: Life safety strategies - Occupant evacuation, behaviour and condition (Sub-system 6). London, 2019.

GOMES, A. G. Sistema de Proteção Contra Incêndio. Rio de Janeiro: Interciência, 1998.

GWYNNE, S. \& KULIGOWSKI, E. D. Skeptic's Discussion on Evacuation Modeling: It's time to challenge the imbalance of security placed on various evacuation models. Fire Protection Engineering, v. 3, n. 3, p, 233-250, 2017. 
JACOBI, P. Cidade e meio ambiente: percepções e práticas em São Paulo, Annablume Editora, São Paulo, 1999.

CAMILO JUNIOR, A. B. Manual de prevenção e combate a incêndios. 15. ed. São Paulo: Senac, 1999. 247 p. ISBN 978-8539603695.

KULIGOWSKI, E. D. Predicting Human Behavior During Fires. Fire Technology, v. 49, p. 101-120, 2013.

KULIGOWSKI, Erica D.; PEACOCK, Richard D.; HOSKINS, Bryan L. A review of building evacuation Models. National Institute of Standards and Technology, U.S., ed. $2^{\mathrm{a}}$, 2010.

LEVIN, B. \& GRONER, N. Some control and communication considerations in designing an emergency elevator evacuation system. The 2nd Symposium on Elevators, Fire and Accessibility. April 19-21, Baltimore, Maryland. p. 190 - 193, 1995.

NELSON, H. E. \& MOWRER, F. W. Emergency Movement. In: SFPE Handbook of Fire Protection Engineering. 3th. ed. Maryland: National Fire Protection Association, 2002.

ONO, R. O impacto do método de dimensionamento das saídas de emergência sobre o projeto arquitetônico: uma análise crítica e proposta de Aprimoramento. 2010. 457 p. Tese (Livre docência em tecnologia da arquitetura). Universidade de São Paulo, São Paulo, 2010.

PEREIRA, A. G. \& POPOVIC, R. R. Tecnologia em Segurança Contra Incêndio. São Paulo: LTR, 2007.

POLÍCIA MILITAR DO ESTADO DE SÃO PAULO. Instrução Técnica n ${ }^{0} 11:$ Saídas de emergência. São Paulo: CBPMESP, 2019.

REIS, Maria do Rosário Frazão. Novas metodologias de evacuação de edifícios - o uso de elevadores. Orientador: João Paulo Correia Rodrigues. 2011. Dissertação (Mestre em Segurança ao Incêndio Urbano) - Universidade de Coimbra, Coimbra, 2011.

REYNOLD, C. W. Steering Behaviors For Autonomous Characters. In: PROCEEDINGS of the Game Developers Conference, 1999. Miller Freeman Game Group, San Francisco, 1999. 
RONCHI, Enrico. Developing and validating evacuation models for fire safety engineering. Fire Safety Journal, [S. I.], 28 abr. 2020.

ARAUJO, J. M. F. Comportamento humano em incêndios. In: SEITO, Alexandre Itiu et al. A segurança contra incêndio no Brasil. São Paulo/SP (Brasil): Projeto Editora, p.93-100, 2008.

SILVA, Valdir Pignatta. Segurança Contra Incêndio em Edifícios Considerações para o Projeto de Arquitetura. 1. ed. São Paulo: Blucher, 2014. v. 1. 129p.

SFPE. Engineering Guide - Human Behavior in Fire. Society of Fire Protection Engineers, 2003.

THUNDERHEAD ENGINEERING. Pathfinder Resources: Technical Reference. Disponível em: $<$ https://www.thunderheadeng.com/pathfinder/resources/>. Acesso em: 16 de junho de 2020.

VALENTIM, M. V. Saídas de emergência em edifícios escolares, 2008. Dissertação (Mestrado em Arquitetura) - Faculdade de Arquitetura, Universidade de São Paulo, 2008. 